\title{
Protect against market exclusivity in the fight against COVID-19
}

\author{
Patents and other intellectual properties could slow down the scale-up and accessibility of \\ treatments and vaccines against COVID-19.
}

W hen a vaccine or a treatment to fight COVID-19 becomes available, the demand will be immediate, enormous and global. The production of such products will need to be on a large scale and will need to preferably take place on different continents and locations.

There is legitimate concern that the pharmaceutical industry will be looking to protect their commercial interests in this crisis, to the detriment of universal access. On 25 March 2020, for instance, Gilead Sciences made the unusual request that the US Food and Drug Administration revoke the orphan drug designation the company had received for remdesivir only 2 days before. The orphan drug designation would have granted Gilead a 7-year market exclusivity in addition to the patent protection it enjoys, and it triggered public criticism. However, remdesivir remains protected by patents until 2029 or 2036 (depending on the claim) in many countries, which will bar competitors from supplying the product unless Gilead offers licenses.

Pricing could also be a barrier to access. Johnson \& Johnson is hoping to develop a vaccine that would reach the market by early 2021 , at an announced price of $€ 10$ per dose. Although this may seem affordable to people in wealthy nations, this price would put a vaccine against COVID-19 out of reach of most low- and middle-income countries. Moreover, it could create market exclusivity for high-income countries that are in a position to hoard the vaccine. This is not a hypothetical situation. During the A/H1N1 influenza pandemic in 2009, wealthy nations negotiated advanced orders of vaccine, to the detriment of developing countries (G. Yamey et al. Lancet https://doi.org/ 10.1016/S0140-6736(20)30763-7; 2020).

Price and production capacity will not be the only determinants of availability. Certain countries may take wartime-like measures and insist that the manufacture and supply of medicines be restricted to domestic use, prohibiting export. This will create problems for the many countries that rely on imports for their drugs, such as the UK's National
Health Service, which imports $80-90 \%$ of its medicines from abroad.

Concerns are not limited to drugs or vaccines. The Netherlands ran into difficulty in scaling up testing for COVID-19 because of a shortage of Roche's lysis buffer-a solution needed in the testing machines of the company that most Dutch hospitals use. Pharmacists would in principle be able to make the solution, but Roche refused to reveal the formula and technical specifications needed to ensure compatibility with Roche's hardware. The Netherlands government, following a fierce debate in its parliament, successfully pressured Roche to release the formula (https://www.dutchnews.nl/news/2020/03/ roche-says-it-has-provided-dutch-with-keycorona-test-recipe/).

In March 2020, some countries took measures against the exploitation of patents and other market exclusivities. Canada and Germany have amended their patent laws to enable swift granting of a compulsory patent license when needed. A compulsory license gives entities other than the patent holder the right to produce, import and sell needed products.

Chile and Ecuador have taken similar measures.

On 19 March 2020, Israel issued compulsory licenses related to lopinavirritonavir to allow the importation of generic lopinavir-ritonavir. AbbVie, the company that holds the patents on the drug, responded swiftly and communicated that it will no longer be enforcing patents related to lopinavir-ritonavir anywhere in the world for any purpose.

The government measures noted above are important for addressing immediate concerns. But they do not address the larger question of how new COVID-19 technologies will go to market and how they will be made available globally. Products for the prevention and treatment of COVID-19 should be global public goods. But saying so does not make it so. That requires action at the global level.

The development of COVID-19 health technologies is currently benefiting from considerable government and charitable funding. By including conditions on public funding, such as upfront agreements on access and affordability, those funding bodies could ensure that all countries can benefit from the innovations.

But a substantial step toward protecting the public-health interest in the COVID-19 pandemic has been recently proposed by the government of Costa Rica. The member state proposed that the WHO establish a global pooling mechanism for patents as well as rights to the data, knowledge and technologies useful in the prevention, detection and treatment of COVID-19.

Unitaid, which finances the Medicines Patent Pool, a United Nations-backed entity that pools patent licenses for the production and supply of essential medicines, has bolstered its investment in the response to COVID-19 and is poised to support the COVID-19 pool proposal (https://unitaid.org/news-blog/ with-special-investment-unitaid-bolstersCOVID-19-response/\#en). The Medicines Patent Pool board announced on 3 April 2020 that it has expanded the mandate of the organization to include any health technology that could contribute to the global response to COVID-19. Other pooling and knowledge-sharing initiatives could be pulled into the effort.

The COVID-19 technology pool should be front and center in the global community's strategy against COVID-19 to ensure that once medicines and vaccines become available, intellectual property will not be the barrier to making them accessible for all in need. The Costa Rican proposal for the COVID-19 technology pool is gaining support. The ball is now in the WHO's court.

\section{Ellen 't Hoen (1) $ه$}

Medicines Law \& Policy, The Netherlands.

凶e-mail: ellenthoen@medicineslawandpolicy.net

Published online: 7 May 2020

https://doi.org/10.1038/s41591-020-0876-6

Competing interests

The author declares no competing interests. 